Cochrane Database of Systematic Reviews

\title{
Medical abortion offered in pharmacy versus clinic-based settings
} (Protocol)

Rodriguez MI, Henderson J, Gartoulla P, Garner P, Edelman A

Rodriguez MI, Henderson J, Gartoulla P, Garner P, Edelman A.

Medical abortion offered in pharmacy versus clinic-based settings.

Cochrane Database of Systematic Reviews 2020, Issue 3. Art. No.: CD013566.

DOI: 10.1002/14651858.CD013566.

www.cochranelibrary.com 
TABLE OF CONTENTS

HEADER 1

ABSTRACT

BACKGROUND

OBJECTIVES

METHODS

ACKNOWLEDGEMENTS

REFERENCES

APPENDICES

CONTRIBUTIONS OF AUTHORS

DECLARATIONS OF INTEREST

SOURCES OF SUPPORT

NOTES 
[Intervention Protocol]

\section{Medical abortion offered in pharmacy versus clinic-based settings}

Maria I Rodriguez ${ }^{1}$, Jillian Henderson ${ }^{1}$, Pragya Gartoulla², Paul Garner ${ }^{3}$, Alison Edelman ${ }^{1}$

1Department of Obstetrics and Gynecology, Oregon Health and Science University, Portland, Oregon, USA. 2 Melbourne School of Population and Global Health, University of Melbourne, Melbourne, Australia. ${ }^{3}$ Department of Clinical Sciences, Liverpool School of Tropical Medicine, Liverpool, UK

Contact address: Maria I Rodriguez, Department of Obstetrics and Gynecology, Oregon Health and Science University, 3181 SW Sam Jackson Park Road, UHN 50, Portland, Oregon, 97239, USA. rodrigma@ohsu.edu.

Editorial group: Cochrane Fertility Regulation Group.

Publication status and date: New, published in Issue 3, 2020.

Citation: Rodriguez MI, Henderson J, Gartoulla P, Garner P, Edelman A. Medical abortion offered in pharmacy versus clinic-based settings. Cochrane Database of Systematic Reviews 2020, Issue 3. Art. No.: CD013566. DOI: 10.1002/14651858.CD013566.

Copyright (c) 2020 The Cochrane Collaboration. Published by John Wiley \& Sons, Ltd.

\section{A B S T R A C T}

This is a protocol for a Cochrane Review (Intervention). The objectives are as follows:

To compare the safety and efficacy of medical abortion offered in pharmacy settings with clinic-based medical abortion. 


\section{B A C K G R O U N D}

\section{Description of the condition}

Unsafe abortion remains a significant threat to women's lives and health (Alkema 2016; Ganatra 2017; WHO 2016). The World Health Organization (WHO) estimates that globally, 25 million unsafe abortions occur every year. Unsafe abortion is the fifth leading cause of maternal mortality (Ganatra 2017).

Improving access to medical abortion is one strategy to reduce unsafe abortion, particularly where trained surgical abortion providers are limited. A growing proportion of abortions globally are medical abortions (Jones 2017; United Nations Population Fund 1994). The WHO has published guidance on effective regimens for medical abortion, and interventions such as laboratory testing or ultrasound are not universally required (WHO 2012).

\section{Description of the intervention}

Medical abortion is offered routinely in clinics and hospitals, but could be offered in other settings such as pharmacies. The safety and effectiveness of medical abortion provision through nonphysician clinicians, such as nurses and auxiliary nurse midwives has been established (Olavarrieta 2015; Warriner 2011). Expanding access to medical abortion through pharmacies is a potential strategy to promote safe abortion care. In many countries, pharmacies are a first and common point of access for women seeking reproductive health information and services, including abortion (Billings 2009; Footman 2018; Sneeringer 2012).

The safety and effectiveness of services obtained from pharmacies relative to other clinical sites are not known. It is possible that lower-quality information or products may be supplied in pharmacies than in clinics, increasing the rates of incomplete abortion or other complications.

Currently, medical abortion is usually offered in clinics and hospitals. Research to date has shown that a medical abortion regimen combining mifepristone with misoprostol is most effective; however, there is some variation in recommendations related to dose, timing and route of administration of the two drugs. A large body of evidence, and recommendations by the WHO, supports the efficacy of a $200 \mathrm{mg}$ dose of mifepristone followed by 800 mcg of misoprostol in pregnancies up to 63 days' gestational age (Raymond 2013; WHO 2014). Recent data support extending its use up to 70 days' gestation (Abbas 2015). These protocols are highly effective and safe, with unsuccessful abortion resulting in approximately 2\% to 5\% of cases (Kulier 2011; Raymond 2013). In settings where mifepristone is not available, medical abortion is carried out using only misoprostol.

The recommended misoprostol regimen is $800 \mu \mathrm{g}$ administered vaginally or sublingually (under the tongue), and repeated at intervals of no less than three hours but no more than 12 hours, for up to three doses. This regimen is $75 \%$ to $90 \%$ effective in completing abortions up to 84 days' gestation. Gestational age is known to affect the efficacy of all regimens, with decreasing efficacy after nine weeks' gestation (Winikoff 2008), which is why regimens for these gestations recommend repeating misoprostol doses. This review will focus on medical abortion provided with mifepristone and misoprostol or misoprostol-alone regimens.

\section{How the intervention might work}

Pharmacies may be able to improve access to safe and effective medical abortion care. Pharmacies are utilized for their convenience, anonymity, and low cost as compared to a traditional health clinic or hospital (Ahmed 2007; Footman 2018). They may improve access for women with limited autonomy, or those living in rural areas, where clinical access is remote (Rocca 2018). Trained pharmacists and pharmacy workers deliver care related to a range of reproductive health conditions, including sexually transmitted infections, emergency contraception and provision of other family planning methods, like birth control pills (Sneeringer 2012). Pharmacists have been successful in delivering reproductive health care because of their ability to provide quick access to necessary information, medications, and referrals, while maintaining confidentiality (Gonsalves 2017).

It is not known how pharmacist provision of medical abortion may impact important safety or efficacy outcomes, as compared with the clinical setting. Pharmacists may have less training than clinicians in accurate usage of the medications to achieve a complete abortion. It is possible that pharmacists working in retail settings would have less time to counsel women than clinicians on known side effects or possible complications, increasing the risk of infection or heavy bleeding leading to hospital attendance. It is important to explore how the setting of care provision (pharmacy versus clinic) impacts key safety and efficacy outcomes, including complete abortion, blood transfusion or hospital admission.

\section{Why it is important to do this review}

Globally, pharmacies play a key role in the formal or informal distribution of information or medications for abortion (Billings 2009; Footman 2018; Lara 2011; Reiss 2016; Reiss 2017; Sneeringer 2012; Tamang 2015; Tamang 2018). Existing data on the safety and efficacy of this practice is limited, and has demonstrated mixed results on the accuracy of information and medical abortion regimens provided by pharmacy workers (Ahmed 2007; Billings 2009; Footman 2018; Reiss 2016; Rocca 2018). Safe and effective abortion can reduce complications associated with unsafe abortion, and maternal mortality (Ganatra 2017; WHO 2012). Pharmacy provision of medical abortion may have the potential to reduce morbidity associated with unsafe abortion. However, evidence is needed to establish whether the safety and effectiveness of care is equivalent to that offered in a clinic.

\section{O B JECTIVES}

To compare the safety and efficacy of medical abortion offered in pharmacy settings with clinic-based medical abortion.

\section{METHODS}

\section{Criteria for considering studies for this review}

\section{Types of studies}

We will seek studies that compare women receiving the same regimen of medical abortion or postabortion care in either a clinic or pharmacy setting. Studies published in any language employing the following designs will be included: randomized trials (clustered or individually randomized); quasi-experimental designs, such as non-randomized or stepped-wedge design experiments; and cohort studies with a control group. 
We will also seek prospective cohort studies that report on outcomes, and compare these between the clinic and pharmacy setting. For safe abortion, programs will incorporate the woman's right to choose her preferred mode of abortion; in other settings, studies may be carried out in countries where abortion access is restricted, and an RCT not possible. Observational studies will reflect programmatic implementation and will detect serious and uncommon harms.

\section{Types of participants}

Pregnant women of any age, seeking abortion care in pharmacies or traditional clinics, are eligible for inclusion.

\section{Types of interventions}

The intervention is pharmacy delivery of any component of medical abortion services. This includes dispensing medical abortion medications. Only studies that provided medical abortion using mifepristone and misoprostol or misoprostol alone will be included. The only administration for mifepristone is oral. Different administration routes and dosing regimens for misoprostol may be used, and we will include studies using any route (oral, sublingual, buccal, and vaginal) or regimen (e.g., repeat dosing). We will consider all types of providers in the intervention group (including pharmacist, pharmacy worker) and in the comparison group (physician, nurse midwife, auxiliary nurse midwife, and nurse). We are not considering surgical abortion outcomes in this review.

\section{Comparison}

Women receiving medical abortion in clinical health care settings compared with pharmacy settings.

\section{Types of outcome measures}

\section{Primary outcomes}

- Complete abortion defined as completion of abortion within 30 days of taking the first medication, and not requiring surgical intervention for completion

- Blood transfusion within 30 days of medical abortion

- Uterine or systemic infection within 30 days of medical abortion

\section{Secondary outcomes}

- Hospital admission for an abortion related event

- Quality of medical abortion care (Any study reported measures of technical or interpersonal quality of care will be considered - priority will be placed on synthesis of validated measures if available; Darney 2018; Darney 2019)

- Additional surgical interventions (besides uterine aspiration)

\section{Search methods for identification of studies}

The Fertility Regulation Group Information Specialist will conduct a search for all published, unpublished, and ongoing studies, without restrictions on language or publication status. The search strategies for each database will be modelled on the search strategy designed for MEDLINE ALL (Ovid) (Epub Ahead of Print, In-Process \& Other Non-Indexed Citations and Daily) in Appendix 1. We will check the bibliographies of included studies and any relevant systematic reviews identified for further references to relevant studies. We will contact experts and organizations in the field to obtain additional information on relevant studies. We may contact original authors for clarification and further data if study reports are unclear. We will consider adverse effects described in included studies only. We will not restrict by language, and will arrange to translate studies published in languages other than English.

\section{Electronic searches}

We will search the following databases from their inception.

- EBM Reviews Ovid - Cochrane Central Register of Controlled Trials

- MEDLINE Ovid (Epub Ahead of Print, In-Process \& Other NonIndexed Citations and Daily)

- Embase.com

- CINAHL

- LILACs http://lilacs.bvsalud.org/en/

- Popline https://www.popline.org/advancedsearch

- Global Health Ovid

- Scopus

We will search the following trials registries.

- The World Health Organization International Clinical Trials Registry Platform www.who.int/trialsearch

- ClinicalTrials.gov www.clinicaltrials.gov

Please see Appendix 1 for the proposed search strategy.

\section{Searching other resources}

We will search the following grey literature sites.

- Guttmacher Institute https://www.guttmacher.org/unitedstates/abortion

- International Planned Parenthood Federation https:// www.ippf.org/

- Ibis Reproductive Health https://ibisreproductivehealth.org/

- Women on Waves https://www.womenonwaves.org/

- Marie Stopes International https://www.mariestopes.org/

- Population Council https://www.popcouncil.org/

- Population Services International https://www.psi.org/

- Ipas https://www.ipas.org/

- Google Scholar https://scholar.google.com/

\section{Data collection and analysis}

\section{Selection of studies}

We will download all titles and abstracts retrieved by electronic searching to a reference management database, and remove duplicates (Covidence). Two reviewers will independently screen titles and abstracts for inclusion. We will retrieve the full-text study reports or publications, and two reviewers will independently screen the full text, identify studies for inclusion, and identify and record reasons for exclusion of the ineligible studies. We will resolve any disagreement through discussion.

We will list studies that initially appeared to meet the inclusion criteria, but that we later excluded, in the 'Characteristics of excluded studies' table. We will collate multiple reports of the same study, so that each study rather than each report is the unit of interest in the review. We will also provide any information we can 
obtain about ongoing studies. We will record the selection process in sufficient detail to complete a PRISMA flow diagram (Liberati 2009).

\section{Data extraction and management}

Two review authors will independently screen and extract data from eligible studies, using a data extraction form designed and pilot-tested by the review authors. We will resolve any disagreements by discussion. Study design and participant characteristics and outcome data will be described in evidence tables. The drugs used, dose, and route of administration will be recorded, as well as each study's inclusion and exclusion criteria.

\section{Assessment of risk of bias in included studies}

Two authors will independently assess any included trials for risk of bias using the Cochrane Risk of Bias Assessment tool (Higgins 2019). We will specifically assess: selection bias (random sequence generation and allocation concealment); performance bias (blinding of participants and personnel); detection bias (blinding of outcome assessors); attrition bias (incomplete outcome data); reporting bias (selective reporting); and other biases. We will pay particular attention to whether there was participant or investigator selection bias towards location of treatment. We will rate studies as low risk, high risk, or unclear risk using the criteria outlined in the Cochrane Handbook for Systematic Reviews of Interventions (Higgins 2019).

For NRS, we will similarly conduct dual, independent assessment of risk of bias using the ROBINS-I tool (Sterne 2016). The domains assessed with this tool are bias at the pre-, at-, and postintervention stages of the study. Specifically, the domains are: pre-intervention bias due to confounding (prognostic variables predict outcome of interest) and selection (inclusion or exclusion of participants related to outcome of interest); at-intervention information bias (misclassification of intervention status); and, post-intervention confounding, selection bias, information bias, and reporting bias. Use of the ROBINS-I tool will facilitate assessment of risk of bias for each domain. For the comparisons we will evaluate, we expect selection bias and confounding preand post-intervention are likely to be of particular importance. The research and clinical expertise of study team members will ensure thorough characterization of risk of bias. We will analyze highquality and low-quality studies in combination and separately to minimize risk of bias. Gestational age is known to be an important confounder in medical abortion (Kahn 2000). The presence of appropriate adjustment for gestational age will be examined in evaluating the risk of bias.

We will use the GRADE approach to assess the quality of the body of evidence used in the meta-analysis for study outcomes. For the synthesis of outcomes drawn from NRS, the evidence will begin with a rating of low certainty given the risks of bias from selection and confounding inherent in NRS designs. We will be using the ROBINS-I tool, however, and may upgrade the certainty level if effects are particularly strong and the risks of confounding and selection are judged to be particularly well-mitigated.

\section{Measures of treatment effect}

For dichotomous data (e.g. complete abortion, yes/no), we will use the number of events in the clinic and pharmacy groups of each study to calculate relative risks (RR) or Mantel-Haenszel odds ratios
(ORs), depending on the most commonly reported effect estimates across the body of evidence. We will extract reported means and standard deviations for continuous outcomes, either as reported in the primary study or calculated from reported estimates of variance to calculate mean difference with $95 \%$ confidence intervals. For NRS, we will prioritize synthesis of study reported adjusted effect estimates and will select the estimate judged to most minimize the risk of bias due to confounding and selection. We will present $95 \%$ confidence intervals as the measure of precision for all outcomes estimates. Where data to calculate ORs, RRs or mean differences are not available, we will use the most detailed numerical data available to facilitate synthesis across included studies (e.g., test statistics, $\mathrm{P}$ values). We will assess whether the estimates we calculate in the review for each individual study are consistent with the available estimates of effects reported in the study publications.

\section{Unit of analysis issues}

The primary unit of analysis will be per woman randomized for RCTs and per woman who undergoes medical abortion (classified as pharmacy or clinic-administered) for NRS. For clusterRCTs included in the review, we will report trial outcome data adjusted for the hierarchical study design (i.e., within cluster correlations among observations that lead to underestimation of standard errors) whenever available and will use these estimates in meta-analysis. Studies that do not report data with appropriate adjustments for study design will be described in tables with the potential for overestimating effect precision noted. For meta-analysis of unadjusted cluster-RCT outcomes, intracluster correlation coefficients (ICC) based on observations from similar studies will be used to estimate adjustments to the standard errors following recommended procedures (Higgins 2019).

\section{Dealing with missing data}

We will analyze the data on an intention-to-treat basis as far as possible. We will reach out to authors to obtain missing data. Where these are unobtainable, we will analyze only the available data.

\section{Assessment of heterogeneity}

We will synthesize effectiveness in a meta-analysis using a randomeffects model, to produce pooled OR, RR, or mean difference effect estimates with $95 \%$ confidence interval $(\mathrm{Cl})$. We are selecting this model a priori to incorporate the effect of trial heterogeneity among prospective studies from different settings. We will assess statistical heterogeneity using the $\mathrm{Chi}^{2}$ tests and $\mathrm{I}^{2}$ statistics. We will use recommendations in the Cochrane Handbook for Systematic Reviews of Interventions to interpret the $1^{2}$ values, and consider a score of over $50 \%$ to indicate the possibility of substantial heterogeneity (Higgins 2019).

\section{Assessment of reporting biases}

Considering the difficulties in detecting and correcting for publication bias and other reporting biases, we will aim to minimize their potential impact by ensuring a comprehensive search for eligible studies, and by being alert for duplication of data. If there are at least 10 studies available for pooled analysis, we will use a funnel plot to explore the possibility of small-study effects.

\section{Data synthesis}

If we judge the studies to be sufficiently similar with respect to study participants, interventions, comparators, and outcomes, effects are 
relatively consistent, and statistical heterogeneity is no greater than moderate $(<60 \%)$ we will conduct meta-analysis using Review Manager 5.3 (Review Manger 2014). We will conduct quantitative synthesis separately for randomized and NRS evidence. We will combine data using the DerSimonian and Laird random-effects model (DerSimonian 1986). These summary effects are grounded in the assumption that the pooled estimate is an average effect from an underlying distribution of true effects. Such a model is appropriate for this body of evidence given the expected heterogeneity of populations and intervention characteristics. For meta-analysis of NRS, we will seek to pool adjusted effect estimates using the generic inverse variance approach. If only unadjusted estimates are available, they will be pooled separately from adjusted effects.

We will present forest plots showing the pooled estimates and $95 \% \mathrm{Cl}$ for each outcome suitable for meta-analysis. Descriptive forest plots for evidence on primary outcomes that are not judged appropriate to pool will also be presented, with a narrative synthesis also provided for outcomes lacking adequate data to combine across studies. This synthesis will consider the consequences of possible incomplete reporting on the outcomes of interest, and the strengths and limitations of available studies for evaluating the review questions.

\section{Subgroup analysis and investigation of heterogeneity}

There may be differences in the effectiveness and safety of pharmacy provided medical abortion depending on several factors, including the following determined $a$ priori to be important to evaluate in subgroup comparisons: Type of health workers providing abortion care in pharmacies (physicians, midwives, nurses, pharmacists, medical assistants), client characteristics (e.g., gestation < 9 weeks, parity), abortion regimen (e.g., dosage, administration route), and the human development index category of the country where the study was conducted.

We will estimate stratified forest plots with pooled subgroup effect estimates and will conduct statistical tests for interaction using meta-regression. If we detect substantial statistical heterogeneity unexplained by these factors, we will explore additional possible explanations in post hoc subgroup analyses derived from the available evidence and its synthesis. When interpreting the results, we will describe the statistical heterogeneity, potential explanatory factors, and inconsistency in the direction of effects across studies that contribute to the pooled effect.

\section{Sensitivity analysis}

As needed, to more fully understand and evaluate the body of evidence, we will conduct sensitivity analyses to assess the effect of risk of bias, removing included studies rated high risk of bias for the primary outcomes.

\section{Summary of findings and assessment of the certainty of the evidence}

We will use GRADEpro and Cochrane methods to prepare a 'Summary of findings' table (GRADEpro GDT; Higgins 2019). The table will evaluate the overall quality of the body of evidence for the review outcomes on effectiveness and safety of medical abortion provided in pharmacy settings. We will use the GRADE criteria (e.g., risk of bias, consistency of effect, imprecision, indirectness, and publication bias) to assess the quality of the evidence (Guyatt 2008). As noted above, the NRS evidence will be initially rated as low quality and the rating will be further refined based on the ROBINSI risk of bias assessments.

Two review authors will work independently to judge the evidence quality (e.g., high, moderate, low, or very low) and will resolve any disagreements by discussion. The reviewers will justify, document, and incorporate their judgments into reporting the results of each outcome.

\section{A C K N OWLE D GEMENTS}

The authors would like to thank the following peer referees who provided comments to improve the protocol: Nada Ata Allah, Philip Darney, and further peer reviewers who wish to remain anonymous, as an exceptional circumstance agreed with the Child and Families Network Senior Editor. The authors would also like to thank Andrea Takeda for copy-editing the protocol. 


\section{RE F E R E N C E S}

\section{Additional references}

\section{Abbas 2015}

Abbas D, Chong E, Raymond EG. Outpatient medical abortion is safe and effective through 70 days gestation. Contraception 2015;92:197-9.

\section{Ahmed 2007}

Ahmed SM, Hossain MA. Knowledge and practice of unqualified and semi-qualified allopathic providers in rural Bangladesh: implications for the HRH problem. Health Policy 2007;84:332-43.

\section{Alkema 2016}

Alkema L, Chou D, Hogan D, Zhang S, Moller A-B, Gemmil A, et al. Global, regional, and national levels and trends in maternal mortality between 1990 and 2015, with scenariobased projections to 2030: a systematic analysis by the UN Maternal Mortality Estimation Inter-Agency Group. Lancet 2016;387:462-74.

\section{Billings 2009}

Billings DL, Walker D, Mainero del Paso G, Clark KA, Dayananda I. Pharmacy worker practices related to use of misoprostol for abortion in one Mexican state. Contraception 2009;79:445-51.

\section{Covidence [Computer program]}

Veritas Health Innovation. Covidence. Version accessed 07 January 2020. Melbourne, Australia: Veritas Health Innovation.

\section{Darney 2018}

Darney BG, Powell B, Anderson K, Baum SE, Blanchard K, Gerdts C, et al. Quality of care and abortion: beyond safety. BMJ Sexual and Reproductive Health 2018;44:159-60.

\section{Darney 2019}

Darney BG, Kapp N, Andersen K, Baum SE, Blanchard K, Gerdts $C$, et al. Definitions, measurement and indicator selection for quality of care in abortion. Contraception 2019;100(5):354-9.

\section{DerSimonian 1986}

DerSimonian R, Laird N. Meta-analysis in clinical trials. Controlled Clinical Trials 1986;7:177-88.

\section{Footman 2018}

Footman K, Keenan K, Reiss K, Reichwein B, Biswas P, Church K. Medical abortion provision by pharmacies and drug sellers in low- and middle-income countries: a systematic review. Studies in Family Planning 2018;49:57-70.

\section{Ganatra 2017}

Ganatra B, Gerdts C, Rossier C, Johnson Jr. BR, Tuncalp O, Assifi A, et al. Global, regional, and subregional classification of abortions by safety 2010-14: estimates from a Bayesian hierarchical model. Lancet 2017;390:2372-81.

\section{Gonsalves 2017}

Gonsalves L, Hindin MJ. Pharmacy provision of sexual and reproductive health commodities to young people: a systematic literature review and synthesis of the evidence. Contraception 2017;95:339-63.

\section{GRADEpro GDT [Computer program]}

McMaster University (developed by Evidence Prime). GRADEpro GDT. Version accessed 13 January 2020. Hamilton (ON): McMaster University (developed by Evidence Prime).

\section{Guyatt 2008}

Guyatt GH, Oxman AD, Vist G, Kunz R, Falck-Ytter Y, AlonsoCoello $P$, et al. GRADE Working Group. GRADE: an emerging consensus on rating quality of evidence and strength of recommendations. BMJ 2008;336(7650):924-6.

\section{Higgins 2019}

Higgins JPT, Thomas J, Chandler J, Cumpston M, Li T, Page MJ, et al (editors). Cochrane Handbook for Systematic Reviews of Interventions version 6.0 (updated July 2019). Cochrane, 2019. Available from www.training.cochrane.org/handbook.

\section{Jones 2017}

Jones RK, Jerman J. Abortion incidence and service availability in the United States, 2014. Perspectives on Sexual and Reproductive Health 2017;49:17-27.

\section{Kahn 2000}

Kahn JG, Becker BJ, Maclsaa L, Amory JK, Neuhaus J, Olkin I, et al. The efficacy of medical abortion: a meta-analysis. Contraception 2000;61:29-40.

\section{Kulier 2011}

Kulier R, Kapp N, Gulmezoglu AM, Hofmeyr GJ, Cheng L, Campana A. Medical methods for first trimester abortion. Cochrane Database of Systematic Reviews 2011, Issue 11. [DOI: 10.1002/14651858.CD002855]

\section{Lara 2011}

Lara D, Garcia SG, Wilson KS, Paz F. How often and under which circumstances do Mexican pharmacy vendors recommend misoprostol to induce an abortion?. International Perspectives on Sexual and Reproductive Health 2011;37:75-83.

\section{Liberati 2009}

Liberati A, Altman DG, Tetzlaff J, Mulrow C, Gøtzsche PC, Ioannidis JP, et al. The PRISMA statement for reporting systematic reviews and meta-analyses of studies that evaluate health care interventions: explanation and elaboration. PLoS Med 2009;6:7.

\section{Olavarrieta 2015}

Olavarrieta CD, Ganatra B, Sorhaindo A, Karver TS, Seuc A, Villalobos A, et al. Nurse versus physician-provision of early medical abortion in Mexico: a randomized controlled noninferiority trial. Bulletin of the World Health Organization 2015;93:249-58. 


\section{Raymond 2013}

Raymond EG, Shannon C, Weaver MA, Winikoff B. First-trimester medical abortion with mifepristone $200 \mathrm{mg}$ and misoprostol: a systematic review. Contraception 2013;87:26-37.

\section{Reiss 2016}

Reiss K, Footman K, Akora V, Liambila W, Ngo TD. Pharmacy workers' knowledge and provision of medication for termination of pregnancy in Kenya. Journal of Family Planning and Reproductive Health Care 2016;42:208-12.

\section{Reiss 2017}

Reiss K, Footman K, Burke E, Diop N, Ndao R, Mane B, et al. Knowledge and provision of misoprostol among pharmacy workers in Senegal: a cross sectional study. BMC Pregnancy Childbirth 2017;17:211.

\section{Review Manger 2014 [Computer program]}

Copenhagen: The Nordic Cochrane Centre, The Cochrane Collaboration. Version 5.3.. Copenhagen: The Nordic Cochrane Centre, The Cochrane Collaboration, 2014.

\section{Rocca 2018}

Rocca CH, Puri M, Shrestha P, Blum M, Maharjan D, Groosman D, et al. Effectiveness and safety of early medication abortion provided in pharmacies by auxiliary nurse-midwives: a noninferiority study in Nepal. PLoS One 2018;13(1):e0191174.

\section{Sneeringer 2012}

Sneeringer RK, Billings DL, Ganatra B, Baird TL. Roles of pharmacists in expanding access to safe and effective medical abortion in developing countries: a review of the literature. Journal of Public Health Policy 2012;33:218-29.

\section{Sterne 2016}

Sterne JA, Hernan MA, Reeves BC, Savovic J, Berkman ND, Viswanathan M, et al. ROBINS-I: a tool for assessing risk of bias in non-randomised studies of interventions. BMJ 2016;355:i4919.

\section{Tamang 2015}

Tamang A, Puri M, Lama K, Shrestha P. Pharmacy workers in Nepal can provide the correct information about using mifepristone and misoprostol to women seeking medication to induce abortion. Reproductive Health Matters 2015;22:104-15.

\section{Tamang 2018}

Tamang A, Puri M, Masud S, Karki DK, Khadka D, Singh M, et al. Medical abortion can be provided safely and effectively by pharmacy workers trained within a harm reduction framework: Nepal. Contraception 2018;97(2):137-43.

\section{United Nations Population Fund 1994}

United Nations Population Fund. Program of Action: Report of the International Conference on Population and Development. Cairo 1994:1-177.

\section{Warriner 2011}

Warriner IK, Wang D, Huong NT, Thapa K, Tamang A, Shah I, et al. Can midlevel health-care providers administer early medical abortion as safely and effectively as doctors? A randomised controlled equivalence trial in Nepal. Lancet 2011;377:1155-61.

\section{WHO 2012}

World Health Organization. Safe abortion: technical and policy guidelines. Second Edition; 2012. Available at: /apps.who.int/ iris/bitstream/handle/10665/70914/9789241548434_eng.pdf.

\section{WHO 2014}

World Health Organization. Clinical practice handbook for safe abortion; 2014. Available at: who.int/reproductivehealth/ publications/unsafe_abortion/clinical-practice-safe-abortion/ en/.

\section{WHO 2016}

World Health Organization. Preventing Unsafe Abortion. Geneva: World Health Organization 2016.

\section{Winikoff 2008}

Winikoff B, Dzuba IG, Creinin MD, Crowden WA, Goldberg AB, Gonzales J, et al. Two distinct oral routes of misoprostol in mifepristone medical abortion: a randomized controlled trial. Obstetrics and Gynecology 2008;112:1303-10.

\section{AP PEN DICES}

\section{Appendix 1. Model search strategy}

\section{Ovid MEDLINE(R) ALL 1946 to 7 January 2020}

Date searched: January 8, 2020

1 Abortion, Induced/ or Abortion, Eugenic/ or Abortion, Legal/ or Abortion, Therapeutic/ or Abortion, Incomplete/ or Abortion Applicants/ or Abortion, Criminal/ or Abortifacient Agents/ or Abortifacient Agents, Nonsteroidal/ or Abortifacient Agents, Steroidal/ or MenstruationInducing Agents/ (42278)

2 (abortifacient ${ }^{\star}$ or abortion* or (menstrua* adj3 regulat $\left.{ }^{\star}\right)$ or pre-abortion or preabortion or post-abortion or postabortion or postabortum or postabortum or feticid* or foeticid ${ }^{\star}$ or ((medical ${ }^{\star}$ or medication or medicin* or trimester $^{\star}$ or gestation* or pregnan $\left.^{\star}\right)$ adj5 $\left(\right.$ post- $^{*}$ terminat* or postterminat $^{\star}$ or pre-terminat* or preterminat $^{\star}$ or terminat $\left.\left.\left.{ }^{\star}\right)\right)\right) . t w, k f .(72951)$

3 Mifepristone/ or Misoprostol/ (9271)

4 (Mifepristone or Misoprostol or Abo-pill or Colestone or Cytotec or Elmif or Epostane or Fenprostalene or GyMiso or Korlym or Medabon or Mefeprin or Mefipil or Mifebort or Mifegest or Mifegyne or Mifeprex or Miferiv or Mifty or Mtpill or Nalador or RU-38486 or RU38486 or RU-486 or RU486 or T-Pill or Termipil).tw,kf. (11441)

5 or/1-4 (95108) 
6 Pharmacies/ or Pharmacists/ or Pharmaceutical Services/ or Pharmacy Technicians/ or Community Pharmacy Services/ (28684) 7 (apothecar ${ }^{\star}$ or chemist* or dispens ${ }^{\star}$ or druggist* or drugstore ${ }^{\star}$ or pharmacy or pharmacies or pharmacist* or OTC or over-the-counter or ((drug or medicine) adj4 (retail ${ }^{\star}$ or seller ${ }^{\star}$ or shop ${ }^{\star}$ or store* or vendor $\left.\left.{ }^{\star}\right)\right)$ ).ti,ab,kf. $(281747)$

8 or/6-7 (287093)

9 and $/ 5,8(650)$

\section{CONTRIBUTIONS OFAUTHORS}

Maria Rodriguez drafted the protocol with input from all authors, and signed off the final protocol for publication.

Jillian Henderson provided input for the draft protocol and signed off the final protocol for publication.

Pragya Gartoulla provided input for the draft protocol and signed off the final protocol for publication.

Paul Garner provided input for the draft protocol and signed off the final protocol for publication.

Alison Edelman provided input for the draft protocol and signed off the final protocol for publication.

\section{DECLARATIONS OF INTEREST}

The authors do not have any interests to declare.

Maria I Rodriguez - nothing to declare.

Jillian Henderson - nothing to declare.

Pragya Gartoulla - nothing to declare.

Paul Garner - nothing to declare.

Alison Edelman - nothing to declare.

\section{SOURCES OF SUPPORT}

\section{Internal sources}

- No internal sources of support, Other.

\section{External sources}

- No external sources of support, Other.

\section{N O TES}

This protocol is based on standard text and guidance provided by the Cochrane Effective Practice and Organisation of Care (EPOC). 